\title{
Determination of glaze thickness on ceramic substrates using an infrared camera
}

\author{
by J. Varis ${ }^{1}$, A. Nurminen², J. Tuominen², A. Autere ${ }^{2}$, and J. Rantala ${ }^{3}$ \\ ${ }^{1}$ Department of Physics, University of Helsinki, FIN-00014 University of Helsinki, Finland; ${ }^{2}$ Department of \\ Computer Science, Helsinki University of Technology, FIN-02150 Espoo, Finland; ${ }^{3}$ Academy of Finland, \\ FIN-00550 Helsinki, Finland
}

\section{Abstract}

The thickness of glaze on a warm ceramic substrate was determined by measuring the surface temperature behaviour of the glaze with an infrared camera. A one-dimensional theoretical model was developed for describing the heat conduction in the glaze. Based on curve fitting of the model into the temperature data, estimates of the glaze thicknesses were obtained. For comparison, the thicknesses were also measured destructively.

\section{Introduction}

Glaze coated ceramic objects like coffee cups, plates, wash basins, etc. are commonplace. The manufacturing process of glaze coated objects is nowadays partly automated and industrial robots handle the spraying of the glaze on the ceramic substrate. Usually, the item is hardened in a furnace after the spraying of the glaze and the quality of the product is examined visually once the product is finished. In the case where the robot spreads too thin a layer on the substrate, the substrate shows through the coating after the hardening process and otherwise complete product has to be rejected. Therefore, the thickness of the layer should be measured non-destructively to reduce raw material waste.

Infrared methods are a possible solution for thickness measurements. Especially, since the substrate material is already heated before it arrives for the glaze spraying and the glaze emulsion itself is cooler than the surrounding air. Contrary to usual coating characterization techniques with active heating[1-4], an infrared detector can now be used for monitoring the temperature behaviour of the sample passively, because a thermal gradient already exists in the sample.

\section{Theoretical model}

The glaze coating can be described one-dimensionally as a solid bound by two infinite plates at $z=0$ and $z=L$ (Figure 1). The plate at $z=O$ represents the boundary between the coating and the substrate, whereas the other plate is the boundary between the glaze and the surrounding air. Assuming, that there is no heat flow from the glaze to the air (including evaporation), that the coating is instantaneously applied, and that the substrate thermal conductivity is much higher than the one of the coating, the boundary conditions for the heat diffusion equation in the coating

$$
\frac{\partial^{2} T(z, t)}{\partial z^{2}}=\frac{1}{\alpha} \frac{\partial T(z, t)}{\partial t}
$$

may be assumed in the form:

$$
\begin{aligned}
& \left.\frac{\partial T(z, t)}{\partial z}\right|_{z=L}=0 \\
& T(0, t)=T_{s} .
\end{aligned}
$$


In Eqs (1) - (3), $T(z, t)$ is the temperature of the coating and $T_{s}$ the temperature of the substrate. $\alpha$ is the thermal diffusivity of the coating. At $t=0$ the substrate temperature $T_{s}$ is higher than that of the coating $T(z, 0)=f(z)$.

With constant diffusivity, the solutions of $\mathrm{Eq}$. (1) for an infinite slab of thickness $L$ can be found for most boundary conditions in Carslaw and Jaeger [4]. However in this case, the coating has water content, that varies in time, and consequently, the diffusivity is also assumed timedependent:

$$
\alpha(t)=a t^{k}+b
$$

$a, b$, and $k$ are constants. The solution of Eq. (1), with conditions (2) - (4), at the glaze surface $(z=L)$ can now be given to have the form

$$
T(L, t)=T_{s^{-}} \frac{4 T_{s}}{\pi} \sum_{n=0}^{\infty} \frac{(-1)^{n}}{2 n+1} \operatorname{Exp}\left[-(2 n+1)^{2} \pi^{2}\left(\frac{a}{k+1} t^{k+1}+b t\right) /\left(4 L^{2}\right)\right],
$$

where the initial temperature of the coating $f(z)$ is considered zero at $t=0$. The constants $a, b$, and $k+1$ can be found by fitting Eq. (5) to the experimental data.

\section{Experimental setup}

The ceramic substrate was hardened in its mold in a furnace. After the mold was removed, the substrate was transfered to the spraying site, where the industrial robot spread the glaze emulsion on the substrate surface. After the spraying, the substrate was once again be transfered to the furnace, where the glaze and the substrate got their final solid form.

The infrared camera was used during the spraying process to monitor the surface temperature of the glaze, before the substrate was conveyed to the furnace the second time (Figure 2). In a typical measurement (Figure 3), a steady temperature followed by rapid temperature fluctuations was observed at first. After a few seconds, a steadily rising surface temperature was observed.

\section{Results}

Figures 4 and 5 show the measured and normalized temperatures for five different glaze thicknesses. Destructively determined thicknesses were $0.23 \mathrm{~mm}, 0.36 \mathrm{~mm}, 0.51 \mathrm{~mm}, 0.70 \mathrm{~mm}$, and $0.80 \mathrm{~mm}$ with an accuracy of $\pm 0.05 \mathrm{~mm}$. A 3-point moving average filter was used for reducing noise in the normalized curves. Figure 5 shows a dependency between the glaze thicknesses and the rise times of the temperature curves. The thinner coatings reach a maximum value faster than the thicker ones, and similarily the temperature starts decaying earlier also. Therefore in principle, the coating thicknesses can be determined based on the temperature measurements.

In Figure 6 the fitted curves are shown in comparison with the experimental data. Eq. (5) was used as the fitted function with $k+1$ having a value of $1 / 2$. Curve fitting was done applying a nonlinear least squares scheme. The curve corresponding to thickness value $0.80 \mathrm{~mm}$ was used for calibrating the destructively obtained values to the fitted ones. The fitted thickness values are shown in Table 1. The thicker values follow the destructively obtained ones reasonably well, but in the thinner values a serious discrepancy is observed. Error estimates for nonlinear fitting techniques are not well defined, but with numerical simulations a rough error of $\pm 15 \%$ can be given for the fitted thickness values. For practical purposes an error limit $\pm 10 \%$ or smaller will be needed. 
Table 1. Destructively measured thickness values vs. thickness values obtained by the curve fitting. The temperature curve with glaze thickness $0.80 \mathrm{~mm}$ was used for calibrating the thickness scale for the fitted values.

\begin{tabular}{cc}
\hline Measured values $(\mathrm{mm})$ & Fitted values $(\mathrm{mm})$ \\
\hline $0.23 \pm 0.05$ & 0.70 \\
0.36 & 0.58 \\
0.51 & 0.59 \\
0.70 & 0.72 \\
0.80 & 0.80 \\
\hline
\end{tabular}

\section{Conclusion}

The measured temperature curves show a dependency between glaze thickness and temperature rise times. Thus in principle, the coating thicknesses can be determined, if the thermal parameters of the material are known. However during the temperature measurement, the water content of the glaze coating, and thermal diffusivity respectively, changes constantly. Since the thermal diffusivity as a function of the water content is not known, absolute values cannot be used for determining the glaze thicknesses at this point. Instead, a curve fitting technique is utilized, and one of the curves is used for calibrating the unknown thermal parameters. The agreement between the fitted thickness values and the destructively measured ones is reasonably good with the larger values, but the smaller values do not coincide. This is probably due to the coating transmission and to the evaporation process that play an important role especially at lower thicknesses, which are not covered by the theoretical model.

\section{Acknowledgements}

Financial support from the Technical Development Centre of Finland and the Chancellor of University of Helsinki is gratefully acknowledged.

\section{REFERENCES}

[1] JAARINEN (J.), LEHTO (A.), and LUUKKALA (M.). - Photothermal measurement of the thickness of diffusion hardened layers in steel. McAvoy (B. R.) ed., Proc. IEEE Symp., 1983, pp. 659-663.

[2] JAARINEN (J.), REYES (C. B.), OPPENHEIM (I. C.), FAVRO (L. D.), KUO (P. K.), and THOMAS (R. L.). - Thermal wave characterization of coated surfaces. Thompson (D. O.) and Chimenti (D. E.) eds., Review of Progress in QNDE vol. 6B, 1987, pp. 1347-1352.

[3] LAU (S. K.), ALMOND (D. P.), and PATEL (P. M.). - Transient thermal wave techniques for the evaluation of surface coatings. J. Phys. D 24, 1991, pp. 428-436.

[4] WU (D.), KARPEN (W.), and BUSSE (G.). - Lockin thermography for multiplex photothermal nondestructive evaluation. Balageas (D.), Busse (G.), and Carlomagno (G. M.) eds., Quantitative Infrared Thermography QIRT ‘92, 1992, pp. 371-376.

[5] CARSLAW (H. S.) and JAEGER (J. C.). - Conduction of Heat in Solids, $2^{\text {nd }}$ Ed. Oxford, Oxford University Press, 510 p., 1959. 


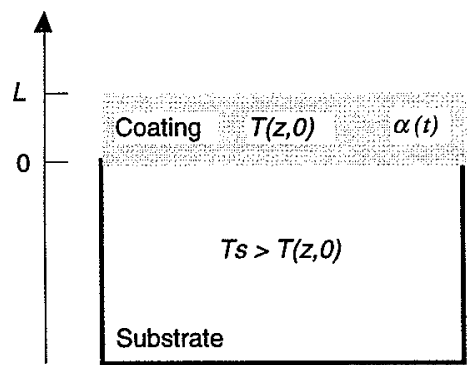

Fig. 1. Theoretical model.

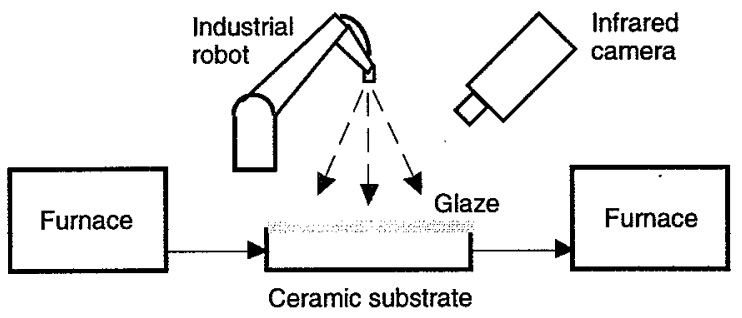

Fig. 2. Measurement setup.

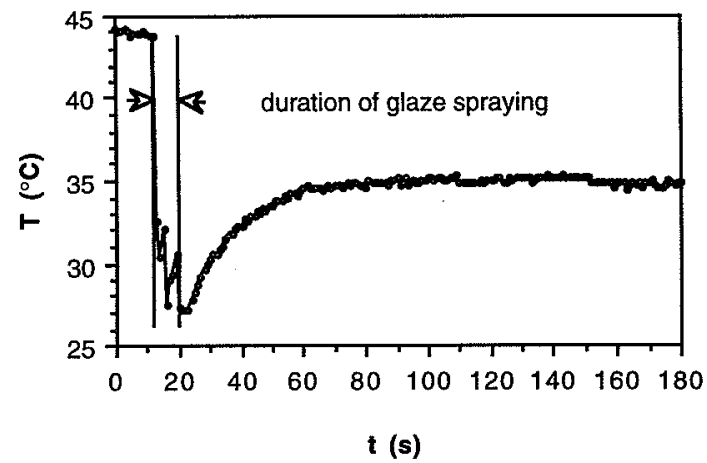

Fig. 3. Typical measured temperature signal. 


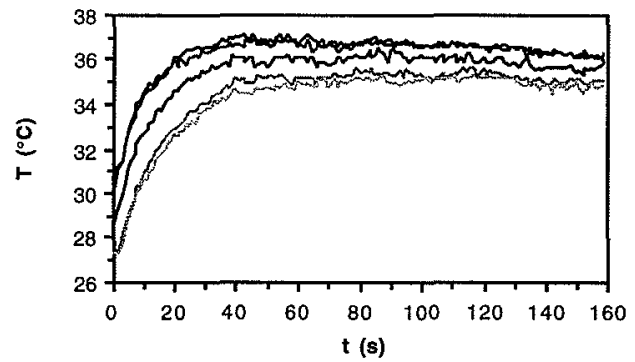

Fig. 4. Measured temperature curves for five different glaze thicknesses. The thicknesses are from top to bottom $0.23 \mathrm{~mm}, 0.36 \mathrm{~mm}, 0.51 \mathrm{~mm}, 0.70 \mathrm{~mm}, 0.80 \mathrm{~mm}$.

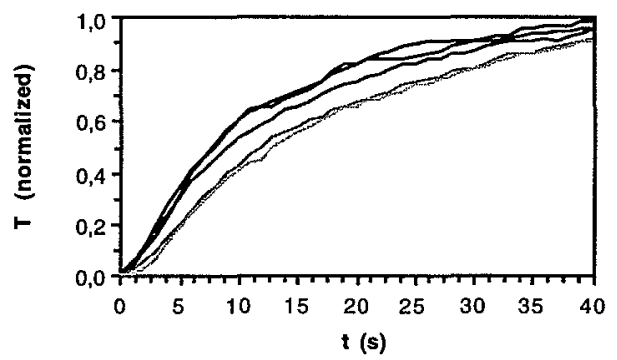

Fig. 5. Normalized temperature curves for the same glaze coatings as in Fig. 4. Notice the different time interval.

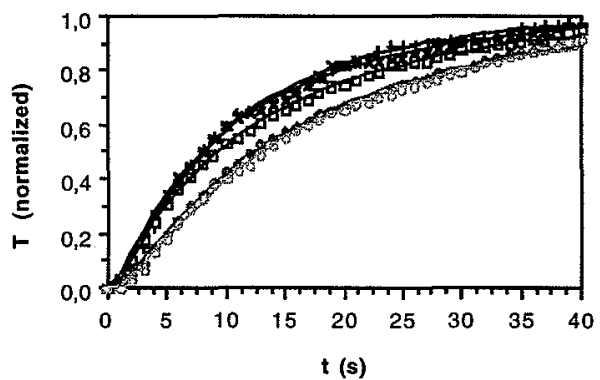

Fig. 6. Fitted temperature curves to the experimental data shown in Fig. 4. 